\title{
Canonical Isomorphism of Two Lie Algebras Arising in $C R$-geometry
}

By

Vladimir V. EzHOV* and Alexander V. ISAEV **

\begin{abstract}
We show that the maximal prolongation of a certain algebra associated with a non-degenerate Hermitian form on $\mathbb{C}^{n} \times \mathbb{C}^{n}$ with values in $\mathbb{R}^{k}$ is canonically isomorphic to the Lie algebra of infinitesimal holomorphic automorphisms of the corresponding quadric in $\mathbb{C}^{n+k}$. This fact creates a link between different approaches to the equivalence problem for Levi-nondegenerate strongly uniform $C R$-manifolds.
\end{abstract}

\section{§0. Introduction and Formulation of Result}

A $C R$-structure on a smooth real manifold $M$ of dimension $m$ is a smooth distribution of subspaces in the tangent spaces $T_{p}^{c}(M) \subset T_{p}(M), p \in M$, with operators of complex structure $J_{p}: T_{p}^{c}(M) \rightarrow T_{p}^{c}(M), J_{p}^{2} \equiv-\mathrm{id}$, that depend smoothly on $p$. A manifold $M$ equipped with a $C R$-structure is called a $C R-$ manifold. It follows that the number $C R \operatorname{dim} M:=\operatorname{dim}_{\mathbb{C}} T_{p}^{c}(M)$ does not depend on $p$; it is called the $C R$-dimension of $M$. The number $C R \operatorname{codim} M:=m-2 C R \operatorname{dim} M$ is called the $C R$-codimension of $M . C R$-structures naturally arise on real submanifolds in complex manifolds. Indeed, if, for example, $M$ is a real submanifold of $\mathbb{C}^{k}$, then one can define the distribution $T_{p}^{c}(M)$ as follows:

$$
T_{p}^{c}(M):=T_{p}(M) \cap i T_{p}(M) .
$$

Communicated by M. Kashiwara, March 30, 1998.

1991 Mathematics Subject Classifications: 32C16, 32F40, 17B66

Keywords and Phrases: $C R$-manifolds, equivalence problem, isomorphism of Lie algebras

*Department of Pure Mathematics, The University of Adelaide, Box 498, G. P. O. Adelaide, South Australia 5001, Australia

e-mail: vezhov@spam.maths.adelaide.edu.au

** Centre for Mathematics and Its Applications, The Australian National University, Canberra, ACT 0200, Australia

e-mail: Alexander.Isaev@anu.edu.au 
On each $T_{p}^{c}(M)$ the operator $J_{p}$ is then defined as the operator of multiplication by $i$. Then $\left\{T_{p}^{c}(M), J_{p}\right\}_{p \in M}$ form a $C R$-structure on $M$, if $\operatorname{dim} \mathbb{C} T_{p}^{c}(M)$ is consiant. This is always the case, for example, if $M$ is a real hypersurface in $\mathbb{C}^{k}$ (in which case $C R \operatorname{codim} M=1$ ). We say that such a $C R$-structure is induced by $\mathbb{C}^{k}$.

A mapping between two $C R$-manifolds $f: M_{1} \rightarrow M_{2}$ is called a $C R$-mapping, if for every $p \in M_{1}$ : (i) $d f(p)$ maps $T_{p}^{c}\left(M_{1}\right)$ to $T_{f(p)}^{c}\left(M_{2}\right)$, and (ii) $d f(p)$ is complex linear on $T_{p}^{c}\left(M_{1}\right)$. Two $C R$-manifolds $M_{1}, M_{2}$ are called $C R$-equivalent, if there is a $C R$-diffeomorphism from $M_{1}$ onto $M_{2}$. Such a $C R$-diffeomorphism $f$ is called a $C R$-isomorphism.

Let $M$ be a $C R$-manifold. For every $p \in M$ consider the complexification $T_{p}^{c}(M) \otimes_{\mathbf{R}}$ C. Clearly, this complexification can be represented as the direct sum

$$
T_{p}^{c}(M) \otimes_{\omega} \mathbb{C}=T_{p}^{(1,0)}(M) \oplus T_{p}^{(0,1)}(M)
$$

where

$$
\begin{aligned}
& T_{p}^{(1,0)}(M):=\left\{X-i J_{p} X: X \in T_{p}^{c}(M)\right\}, \\
& T_{p}^{(0,1)}(M):=\left\{X+i J_{p} X: X \in T_{p}^{c}(M)\right\} .
\end{aligned}
$$

The $C R$-structure on $M$ is called integrable if for any local sections $Z, Z^{\prime}$ of the bundle $T^{(1,0)}(M)$, the vector field $\left[Z, Z^{\prime}\right]$ is also a section of $T^{(1,0)}(M)$. It is not difficult to see that if $M \subset \mathbb{C}^{K}$ and the $C R$-structure on $M$ is induced by $\mathbb{C}^{K}$, then it is integrable.

An important characteristic of a $C R$-structure called the Levi form comes from taking commutators of local sections of $T^{(1,0)}(M)$ and $T^{(0,1)}(M)$. Let $p \in M$, $z, z^{\prime} \in T_{p}^{(1,0)}(M)$, and $Z, Z^{\prime}$ be local sections of $T^{(1,0)}(M)$ near $p$ such that $Z(p)=$ $z, Z^{\prime}(p)=z^{\prime}$. The Levi form of $M$ at $p$ is the Hermitian form on $T_{p}^{(1,0)}(M) \times T_{p}^{(1,0)}$ with values in $\left(T_{p}(M) / T_{p}^{c}(M)\right) \bigotimes_{\mathbb{B}} \mathbb{C}$ given by

$$
\mathscr{L}_{M}(p)\left(z, z^{\prime}\right):=i\left[Z, \bar{Z}^{\prime}\right](p)\left(\bmod T_{p}^{c}(M) \bigotimes_{\mathbb{R}} \mathbb{C}\right) .
$$

The Levi form is defined uniquely up to the choice of coordinates in $\left(T_{p}(M) /\right.$ $\left.T_{p}^{c}(M)\right) \otimes_{\mathbb{R}} \mathbb{C}$, and, for fixed $z$ and $z^{\prime}$, its value does not depend on the choice of $Z$ and $Z^{\prime}$

Let $H=\left(H^{1}, \cdots, H^{k}\right)$ be a Hermitian form on $\mathbb{C}^{n} \times \mathbb{C}^{n}$ with values in $\mathbb{R}^{k}$. For any such $H$ there is a corresponding standard $C R$-manifold $Q_{H} \subset \mathbb{C}^{n+k}$ of $C R$-dimension $n$ and $C R$-codimension $k$ defined as follows:

$$
Q_{H}:=\{(z, w): \operatorname{Im} w=H(z, z)\},
$$

where $z:=\left(z_{1}, \cdots, z_{n}\right), w:=\left(w_{1}, \cdots, w_{k}\right)$ are coordinates in $\mathbb{C}^{n+k}$. The manifold $Q_{H}$ is often called the quadric associated with the form $H$. The Lcvi form of $Q_{H}$ at any point is given by $H$. 
A Hermitian form $H$ is called non-degenerate if:

(i) The scalar Hermitian forms $H^{1}, \cdots, H^{k}$ are linearly independent over $\mathbb{R}$;

(ii) $H\left(z, z^{\prime}\right)=0$ for all $z^{\prime} \in \mathbb{C}^{n}$ implies $z=0$.

A $C R$-structure on $M$ is called Levi non-degenerate, if its Levi form at any $p$ $\in M$ is non-degenerate. An important tool in the geometry of Levi non-degenerate integrable $C R$-manifolds is the automorphism group of $Q_{H}$. Let Aut $\left(Q_{H}\right)$ denote the collection of all local $C R$-isomorphisms of $Q_{H}$ to itself that we call local $C R$-automorphisms. It turns out that, if $H$ is non-degenerate, then any local $C R$-automorphism extends to a rational (more precisely, a matrix fractional quadratic) map of $\mathbb{C}^{n+k}[8],[7],[12]$, [5]. Thus, for a nondegenerate $H$, Aut $\left(Q_{H}\right)$ is a finite-dimensional Lie group. Let $\mathfrak{g}_{H}$ denote the Lie algebra of Aut $\left(Q_{H}\right)$. As shown in [1], [10], (see also [6] for a simple proof), the algebra $\mathfrak{g}_{H}$ consists of polynomial vector fields on $\mathbb{C}^{n+k}$ of the form

$$
\begin{aligned}
\mathfrak{g}_{H}= & \left\{(p+C z+a w+A(z, z)+B(z, w)) \frac{\partial}{\partial z}+\right. \\
& \left.(q+2 i H(z, p)+s w+2 i H(z, a \bar{w})+r(w, w)) \frac{\partial}{\partial w}\right\},
\end{aligned}
$$

where $p \in \mathbb{C}^{n}, q \in \mathbb{R}^{k}, C$ is an $n \times n$-matrix, $s$ is a $k \times k$-matrix, $A(z, z)$ is a quadratic form on $\mathbb{C}^{n} \times \mathbb{C}^{n}$ with values in $\mathbb{C}^{n}, a$ is an $n \times k$-matrix, $B(z, w)$ is a bilinear form on $\mathbb{C}^{n} \times \mathbb{C}^{k}$ with values in $\mathbb{C}^{n}, r\left(w_{1}, w_{2}\right)$ is a symmetric bilinear form on $\mathbb{C}^{k} \times \mathbb{C}^{k}$ with values in $\mathbb{C}^{k}$, and the following holds

$$
\begin{aligned}
2 \operatorname{Re} H(C z, z) & =s H(z, z), \\
H(A(z, z), z) & =2 i H(z, a H(z, z)), \\
\operatorname{Re} H(B(z, u), z) & =r(H(z, z), u), \\
\operatorname{Im} H(B(z, H(z, z)), z) & =0,
\end{aligned}
$$

for all $z \in \mathbb{C}^{n}, u \in \mathbb{R}^{k}$.

We can now make $g_{H}$ into a graded Lie algebra by introducing weights as follows: $z$ has weight $1, w$ has weight $2, \frac{\partial}{\partial z}$ has weight $-1, \frac{\partial}{\partial w}$ has weight -2 . Then we get $g_{H}=\bigoplus_{l=-2}^{2} g_{H}^{l}$, where

$$
\begin{aligned}
g_{H}^{-2}: & =\left\{q \frac{\partial}{\partial w}\right\}, \\
\mathfrak{g}_{H}^{-1} & =\left\{p \frac{\partial}{\partial z}+2 i H(z, p) \frac{\partial}{\partial w}\right\}, \\
\mathfrak{g}_{H}^{0}: & =\left\{C z \frac{\partial}{\partial z}+s w \frac{\partial}{\partial w}\right\}, \\
\mathfrak{g}_{H}^{1} & :=\left\{(a w+A(z, z)) \frac{\partial}{\partial z}+2 i H(z, a \bar{w}) \frac{\partial}{\partial w}\right\}, \\
g_{H}^{2} & :=\left\{B(z, w) \frac{\partial}{\partial z}+r(w, w) \frac{\partial}{\partial w}\right\} .
\end{aligned}
$$


Note that $Q_{H}$ is a homogeneous manifold since the global $C R$-automorphisms

$$
\begin{aligned}
& z^{\mapsto} z+z^{0}, \\
& w \mapsto w+w^{0}+2 i H\left(z, z^{0}\right),
\end{aligned}
$$

for $\left(z^{0}, w^{0}\right) \in Q_{H}$, act transitively on $Q_{H}$. The subalgebra $\mathfrak{g}_{H}^{-1} \oplus \mathfrak{g}_{H}^{-2}$ is the Lie algebra of the subgroup of $\operatorname{Aut}\left(Q_{H}\right)$ consisting of automorphisms of the form (0.4). The subalgebra $g_{H}^{0}$ is the Lie algebra of the subgroup of Aut $\left(Q_{H}\right)$ consisting of linear automorphisms, i.e. automorphisms of the form

$$
z^{\mapsto} \mapsto P z, \quad w \mapsto R w,
$$

where $P$ is a complex $n \times n$-matrix, $R$ is a real $k \times k$-matrix such that

$$
R^{-1} H(P z, P z)=H(z, z) .
$$

The components $\mathrm{g}_{H}^{1}, \mathrm{~g}_{H}^{2}$ are responsible for the existence of nonlinear automorphisms of $Q_{H}$ that preserve the origin.

An example of how the algebra $g_{H}$ is used in $C R$-geometry is the equivalence problem for strongly uniform $C R$-manifolds. Let $H_{1}, H_{2}$ be two $\mathbb{R}^{k}$-valued Hermitian forms on $\mathbb{C}^{n} \times \mathbb{C}^{n}$. We say that $H_{1}$ and $H_{2}$ are equivalent, if there exist linear transformations $A$ of $\mathbb{C}^{n}$ and $B$ of $\mathbb{R}^{k}$ such that

$$
H_{2}(z, z)=B H_{1}(A z, A z) \text {. }
$$

We call a $C R$-manifold $M$ strongly uniform, if the forms $\mathscr{L}_{M}(p)$ are equivalent for all $p \in M$. If, for example, $M$ is Levi non-degenerate and $C R$ codim $M=1$ then $M$ is strongly uniform. The equivalence problem for strongly uniform Levi non-degenerate integrable $C R$-manifolds is usually approached by constructing a $C R$-invariant parallelism on certain bundles over the manifolds with values in a suitable Lie algebra. In a number of cases (see [2], [3], [9], [4]) this Lie algebra was chosen to be $g_{H}$, where $H$ is a Hermitian form equivalent to any $\mathscr{L}_{M}(p), p \in M$. In the general approach of Tanaka [11], however, a seemingly different algebra was used: Tanaka considered a certain maximal prolongation $\widetilde{\mathfrak{g}}_{H}$ of $\mathfrak{g}_{H}^{-2} \oplus \mathrm{g}_{H}^{-1} \oplus \mathfrak{g}_{H}^{0}$. It is therefore a reasonable question whether the algebras $\mathrm{g}_{H}$ and $\widetilde{\mathrm{g}}_{H}$ are isomorphic. In this paper we give a positive answer to this question in the main theorem below (see [11] [9], [4] for partial results).

We will now give the precise definition of the algebra $\widetilde{\mathfrak{g}}_{H}$ from [11]. It is defined as an a priori infinite-dimensional graded Lie algebra

$$
\tilde{\mathrm{g}}_{H}=\mathrm{g}_{H}^{-2} \oplus \mathrm{g}_{H}^{-1} \oplus \mathrm{g}_{H}^{0} \oplus\left(\bigoplus_{l=1}^{\infty} \widetilde{\mathrm{g}}_{H}^{l}\right)
$$

which is maximal among all Lie algebras of the above form that satisfy the conditioons:

(i) For $l \geq 0$ and $X \in \widetilde{\mathfrak{g}}_{H}^{l},\left[X, \mathfrak{g}_{H}^{-1}\right]=0$ implies $X=0$; 


\section{(ii) $\mathfrak{g}_{H}^{-2} \oplus \mathfrak{g}_{H}^{-1} \oplus \mathfrak{g}_{H}^{0}$ is a subalgebra of $\tilde{\mathfrak{g}}_{H}$.}

It is shown in [11] that $\widetilde{\mathfrak{g}}_{H}$ is unique and can be constructed by the following inductive procedure. First we define vector spaces $\tilde{\mathfrak{g}}_{H}^{l}$ and brackets $\left[X_{l}, X_{-1}\right] \in \widetilde{\mathfrak{g}}_{H}^{l-1},\left[X_{l}, X_{-2}\right] \in \tilde{\mathfrak{g}}_{H}^{l-2}$, where $X_{p} \in \tilde{\mathfrak{g}}_{H}^{l}$ (we set $\tilde{\mathrm{g}}_{H}^{l}:=\mathrm{g}_{H}^{l}$ for $l=-2,-1$, $0)$. Suppose that these spaces and brackets have been defined for $0 \leq l \leq L-1$ in such a way that the following holds

$$
\begin{aligned}
{\left[\left[X_{l}, X_{-1}\right], Y_{-1}\right]-\left[\left[X_{l}, Y_{-1}\right], X_{-1}\right] } & =\left[X_{l},\left[X_{-1}, Y_{-1}\right]\right], \\
{\left[\left[X_{l}, X_{-2}\right], X_{-1}\right] } & =\left[\left[X_{l}, X_{-1}\right], X_{-2}\right],
\end{aligned}
$$

for all $X_{l} \in \widetilde{\mathfrak{g}}_{H}^{l}, X_{-1}, Y_{-1} \in \widetilde{\mathfrak{g}}_{H}^{-1}$. Then we define $\widetilde{\mathfrak{g}}_{H}^{L}$ to be the vector space of all linear mappings $X_{L}: \widetilde{\mathfrak{g}}_{H}^{-1} \rightarrow \widetilde{\mathfrak{g}}_{H}^{L-1}$ for which there exist linear mappings $X_{L}^{\prime}: \widetilde{\mathfrak{g}}_{H}^{-2} \rightarrow$ $\tilde{\mathfrak{g}}_{H}^{L-2}$ such that

$$
\begin{aligned}
{\left[X_{L}\left(X_{-1}\right), Y_{-1}\right]-\left[X_{L}\left(Y_{-1}\right), X_{-1}\right] } & =X_{L}^{\prime}\left(\left[X_{-1}, Y_{-1}\right]\right), \\
{\left[X_{L}^{\prime}\left(X_{-2}\right), X_{-1}\right] } & =\left[X_{L}\left(X_{-1}\right), X_{-2}\right],
\end{aligned}
$$

for all $X_{-1}, Y_{-1} \in \widetilde{\mathfrak{g}}_{H}^{-1}, X_{-2} \in \widetilde{\mathfrak{g}}_{H}^{-2}$. We set $\left[X_{L}, X_{-1}\right]:=X_{L}\left(X_{-1}\right)$ for all $X_{-1} \in \widetilde{\mathfrak{g}}_{H}^{-1}$. Since $H$ is non-degenerate, we have $\tilde{\mathrm{g}}_{H}^{-2}=\left[\tilde{\mathrm{g}}_{H}^{-1}, \tilde{\mathrm{g}}_{H}^{-1}\right]$, and therefore $X_{L}^{\prime}$ is uniquely determined by $X_{L}$. Then we set $\left[X_{L}, X_{-2}\right]:=X_{L}^{\prime}\left(X_{-2}\right)$ for all $X_{-2} \in \widetilde{\mathfrak{g}}_{H}^{-2}$. We also set $\left[X_{-1}, X_{l}\right]:=-\left[X_{l}, X_{-1}\right]$ and $\left[X_{-2}, X_{l}\right]:=-\left[X_{l}, X_{-2}\right]$. Clearly, $(0.6)$ then gives equations $(0.5)$ for $l=L$.

Note that equations $(0.5)$ imply

$$
\left[\left[X_{l}, X_{-2}\right], Y_{-2}\right]=\left[\left[X_{l}, Y_{-2}\right], X_{-2}\right] \text {, }
$$

for all $X_{l} \in \widetilde{\mathfrak{g}}_{H}^{l}, l \geq 0$, and $X_{-2}, Y_{-2} \in \widetilde{\mathfrak{g}}_{H}^{-2}$.

Let us now define brackets $\left[X_{p}, X_{q}\right] \in \tilde{\mathrm{g}}_{H}^{p+q}, X_{p} \in \tilde{\mathrm{g}}_{H}^{t}, X_{q} \in \tilde{\mathrm{g}}_{H}^{D}, p, q \geq 0$, inductively as follows. Suppose that these brackets have been defined for $p, q \geq$ $0, p+q \leq L-1$, in such a way that for any $X_{p} \in \widetilde{\mathfrak{g}}_{H}^{q}, X_{q} \in \widetilde{\mathfrak{g}}_{H}^{q}$ the following holds

$$
\begin{aligned}
& {\left[\left[X_{p}, X_{q}\right], X_{-1}\right]=\left[\left[X_{p}, X_{-1}\right], X_{q}\right]+\left[X_{p},\left[X_{q}, X_{-1}\right]\right],} \\
& {\left[\left[X_{p}, X_{q}\right], X_{-2}\right]=\left[\left[X_{p}, X_{-2}\right], X_{q}\right]+\left[X_{p},\left[X_{q}, X_{-2}\right]\right],}
\end{aligned}
$$

for all $X_{-1} \in \widetilde{\mathfrak{g}}_{H}^{-1}, X_{-2} \in \widetilde{\mathfrak{g}}_{H}^{-2}$. We take any $X_{p} \in \widetilde{\mathfrak{g}}_{H}^{p}, X_{q} \in \widetilde{\mathfrak{g}}_{H}^{q}$ with $p, q \geq 0$ and $p+q=$ $L$ and define linear mappings $X_{L}$ and $X_{L}^{\prime}$ from $\widetilde{\mathfrak{g}}_{H}^{-1}$ and $\widetilde{\mathfrak{g}}_{H}^{-2}$ to $\widetilde{\mathfrak{g}}_{H}^{L-1}$ and $\tilde{\mathfrak{g}}_{H}^{L-2}$ respectively by

$$
\begin{aligned}
& X_{L}\left(X_{-1}\right):=\left[\left[X_{p}, X_{-1}\right], X_{q}\right]+\left[X_{p},\left[X_{q}, X_{-1}\right]\right], \\
& X_{L}^{\prime}\left(X_{-2}\right):=\left[\left[X_{p}, X_{-2}\right], X_{q}\right]+\left[X_{p},\left[X_{q}, X_{-2}\right]\right] .
\end{aligned}
$$

Then we see that $X_{L}, X_{L}^{\prime}$ so defined satisfy (0.6) and therefore $X_{L} \in \widetilde{\mathrm{g}}_{H}^{L}$. We then define $\left[X_{p}, X_{q}\right]:=X_{L}$. Clearly, this definition gives identities (0.8) for all $p, q \geq$ $0, p+q=L$. Thus $\left[X_{p}, X_{q}\right]$ have been defined for all $p, q \geq 0$. Note that $\left[X_{p}, X_{q}\right]=$ 
$-\left[X_{q}, X_{p}\right]$ for all $p, q \geq 0$. By induction, we can also prove

$$
\left[\left[X_{p}, X_{q}\right], X_{r}\right]+\left[\left[X_{q}, X_{r}\right], X_{p}\right]+\left[\left[X_{r}, X_{p}\right], X_{q}\right]=0,
$$

for all $X_{p} \in \widetilde{\mathfrak{g}}_{H}^{q}, X_{q} \in \widetilde{\mathfrak{g}}_{H}^{q}, X_{r} \in \widetilde{\mathfrak{g}}_{H}^{r}, p, q, r \geq 0$. By (0.5), (0.7), (0.8), (0.9) the brackets defined above give a Lie algebra structure on $\widetilde{\mathfrak{g}}_{H}$. This completes the construction of $\widetilde{\mathfrak{g}}_{H}$ in [11].

We now define a mapping $\bar{\Phi}: \mathfrak{g}_{H} \rightarrow \widetilde{\mathfrak{g}}_{H}$ as follows:

$$
\begin{aligned}
& \bar{\Phi} \text { is identical on } \mathfrak{g}_{H}^{-2} \oplus \mathfrak{g}_{H}^{-1} \oplus \mathfrak{g}_{H}^{0}, \\
& {[\Phi(X)](X-1):=\left[X, X_{-1}\right] \text { for } X \in \mathfrak{g}_{H}^{1} .} \\
& {\left[[\Phi(X)]\left(X_{-1}\right)\right]\left(Y_{-1}\right):=\left[\left[X, X_{-1}\right], Y_{-1}\right] \text { for } X \in \mathrm{g}_{H}^{2} .}
\end{aligned}
$$

It follows that $\Phi$ is a Lie algebra homomorphism and ker $\Phi=\{0\}$. Moreover, $\Phi\left(\mathfrak{g}_{H}^{b}\right) \subset \widetilde{\mathfrak{g}}_{H}^{b}$ for $p=1,2$.

We are now ready to formulate the main result of the paper.

Theorem 0.1. The mapping $\Phi$ is an isomorphism.

We will prove the theorem in the next section. Before proceeding, we would like to thank G. Schmalz for useful discussions.

\section{\$1. Proof of Theorem}

It is clear from the preceding discussion that to prove the theorem it is sufficient to show that $\operatorname{dim} \mathrm{g}_{H}^{\phi}=\operatorname{dim} \widetilde{\mathrm{g}}_{H}^{t}$ for $p=1,2$, and $\widetilde{\mathrm{g}}_{H}^{3}=\{0\}$.

Lemma 1.1. $\operatorname{dim} \mathrm{g}_{H}^{1}=\operatorname{dim} \tilde{\mathrm{g}}_{H}^{1}$.

Proof. Let $X_{1} \in \tilde{\mathfrak{g}}_{H}^{1}$. Since $\mathfrak{g}_{H}^{-1}, \mathfrak{g}_{H}^{0}$ are given in the form as in $(0.3 . \mathrm{b}),(0.3 . c)$, $X_{1}$ can be written as

$$
X_{1}\left(p \frac{\partial}{\partial z}+2 i H(z, p) \frac{\partial}{\partial w}\right)=\phi(p) \frac{\partial}{\partial z}+\phi(p) w \frac{\partial}{\partial w},
$$

$p \in \mathbb{C}^{n}$, where $\phi, \phi$ are real-linear mapppings from $\mathbb{C}^{n}$ to the spaces $M(n, \mathbb{C})$ of complex $n \times n$ - and $M(k, \mathbb{R})$ of real $k \times k$-matrices respectively such that, for any $p, z \in \mathbb{C}^{n}$,

$$
\phi(p) H(z, z)=2 \operatorname{Re} H(\phi(p) z, z)
$$

(see $\left(0.2\right.$.a)). Let $X_{1}^{\prime}$ be the linear mapping from $\mathfrak{g}_{H}^{-2}$ to $\mathfrak{g}_{H}^{-1}$ corresponding to $X_{1}$ as in the definition of $\tilde{\mathfrak{g}}_{H}^{-1}$. It then follows from $(0.3 . \mathrm{a}),(0.3 . \mathrm{b})$ that $X_{1}^{\prime}$ can be written in the form

$$
X_{1}^{\prime}\left(q \frac{\partial}{\partial w}\right)=\mu(q) \frac{\partial}{\partial z}+2 i H(z, \mu(q)) \frac{\partial}{\partial w},
$$


$q \in \mathbb{R}^{k}$, where $\mu$ is a linear mapping from $\mathbb{R}^{k}$ to $\mathbb{C}^{n}$. Next, conditions $(0.6)$ for $L$ $=1$ are equivalent to

$$
\begin{aligned}
& 4 \mu\left(\operatorname{Im} H\left(p_{1}, p_{2}\right)\right)=\phi\left(p_{1}\right) p_{2}-\phi\left(p_{2}\right) p_{1}, \\
& 4 \operatorname{Im} H(\mu(q), p)=\phi(p) q,
\end{aligned}
$$

for all $p, p_{1}, p_{2} \in \mathbb{C}^{n}, q \in \mathbb{R}^{k}$.

We set

$$
A(p, p):=\frac{1}{2} \phi(p) p-i \mu(H(p, p)), \quad a:=\mu
$$

$p \in \mathbb{C}^{n}$. We will show that the following holds (cf. (0.2.b))

$$
H(A(p, p), p)=2 i H(p, a H(p, p)),
$$

for all $p \in \mathbb{C}^{n}$. We write $\phi$ in the most general form

$$
\phi(p)=R p+Q \bar{p},
$$

where $R, Q$ are constant vectors of length $n$ with entries from $M(n, \mathbb{C})$. Formulas (1.1), (1.2) then give

$$
\begin{aligned}
R p_{1} p_{2} & =R p_{2} p_{1} \\
Q \bar{p}_{1} p_{2} & =2 i \mu\left(H\left(p_{2}, p_{1}\right)\right), \\
H\left(R p_{1} p_{2}, p_{3}\right) & =2 i H\left(p_{1}, \mu\left(H\left(p_{2}, p_{3}\right)\right)\right)-H\left(p_{2}, Q \bar{p}_{1} p_{3}\right),
\end{aligned}
$$

for all $p_{1}, p_{2}, p_{3} \in \mathbb{C}^{n}$, where $\mu$ is complex-linearly extended from $\mathbb{R}^{k}$ to $\mathbb{C}^{k}$. Identities (1.3) easily follow from $(1.4 . \mathrm{b}),(1.4 . \mathrm{c})$. Identity $(1.4 . \mathrm{b})$ in addition gives

$$
A(p, p)=\frac{1}{2} R p p
$$

thus showing that $A$ is a quadratic form on $\mathbb{C}^{n} \times \mathbb{C}^{n}$.

It is clear from (1.1), (1.4.b), (1.4.c) that $a$ uniquely determines $X_{1}$ (also note that (1.3) implies that $A$ and $a$ uniquely determine each other), and the lemma is proved.

Lemma 1.2. $\operatorname{dimg} g_{H}^{2}=\operatorname{dim} \tilde{\mathrm{g}}_{H}^{2}$.

Proof. Let $X_{2} \in \widetilde{\mathfrak{g}}_{H}^{2}$. It follows from (0.3.a) - (0.3.c) that there exist real-bilinear mappings $\phi(\cdot, \cdot)$ and $\phi(\cdot, \cdot)$ from $\mathbb{C}^{n} \times \mathbb{C}^{n}$ to the spaces $M(n$, $\mathbb{C})$ and $M(k, \mathbb{R})$ respectively, and a real-bilinear mapping $\mu(\cdot, \cdot)$ from $\mathbb{C}^{n} \times$ $\mathbb{R}^{k}$ to $\mathbb{C}^{n}$ such thaat

$$
\begin{gathered}
X_{2}\left(p_{1} \frac{\partial}{\partial z}+2 i H\left(z, p_{1}\right) \frac{\partial}{\partial w}\right)\left(p_{2} \frac{\partial}{\partial z}+2 i H\left(z, p_{2}\right) \frac{\partial}{\partial w}\right)=\phi\left(p_{1}, p_{2}\right) z \frac{\partial}{\partial z}+\phi\left(p_{1}, p_{2}\right) w \frac{\partial}{\partial w}, \\
{\left[X_{2}\left(p \frac{\partial}{\partial z}+2 i H(z, p) \frac{\partial}{\partial w}\right)\right]^{\prime}\left(q \frac{\partial}{\partial w}\right)=\mu(p, q) \frac{\partial}{\partial z}+2 i H(z, \mu(p, q)) \frac{\partial}{\partial w},}
\end{gathered}
$$


$p, p_{1}, p_{2} \in \mathbb{C}^{n}, q \in \mathbb{R}^{k}$, where $\left[X_{2}\left(p \frac{\partial}{\partial z}+2 i H(z, p) \frac{\partial}{\partial w}\right)\right]^{\prime}$ corresponds to $X_{2}\left(p \frac{\partial}{\partial z}+\right.$ $\left.2 i H(z, p) \frac{\partial}{\partial w}\right)$ as an element of $\tilde{\mathrm{g}}_{H}^{\mathrm{l}}$. Let $X_{2}^{\prime}$ be the corresponding linear mapping from $\mathrm{g}_{H}^{-2}$ to $\mathrm{g}_{H}^{0}$. It follows from $(0.3 . \mathrm{a}),(0.3 . \mathrm{c})$ that it can be written in the form

$$
X_{2}^{\prime}\left(q \frac{\partial}{\partial w}\right)=\eta(q) z \frac{\partial}{\partial z}+\nu(q) w \frac{\partial}{\partial w}
$$

$q \in \mathbb{R}^{k}$, where $\eta$ and $\nu$ are linear mappings from $\mathbb{R}^{k}$ to the spaces $M(n, \mathbb{C})$ and $M(k, \mathbb{R})$ respectively. Equation (0.2.a) gives that the following conditions are satisfied

$$
\begin{aligned}
\phi\left(p_{1}, p_{2}\right) H(z, z) & =2 \operatorname{Re} H\left(\phi\left(p_{1}, p_{2}\right) z, z\right), \\
\nu(q) H(z, z) & =2 \operatorname{Re} H(\eta(q) z, z),
\end{aligned}
$$

for all $p_{1}, p_{2}, z \in \mathbb{C}^{n}, q \in \mathbb{R}^{k}$. Next, analogously to (1.2), the following holds

$$
\begin{aligned}
4 \mu\left(p_{1}, \operatorname{Im} H\left(p_{2}, p_{3}\right)\right) & =\phi\left(p_{1}, p_{2}\right) p_{3}-\phi\left(p_{1}, p_{3}\right) p_{2}, \\
4 \operatorname{Im} H\left(\mu\left(p_{1}, q\right), p_{2}\right) & =\phi\left(p_{1}, p_{2}\right) q,
\end{aligned}
$$

for all $p, p_{1}, p_{2} \in \mathbb{C}^{n}, q \in \mathbb{R}^{k}$. Further, conditions (0.6) for $L=2$ are equivalent to

$$
\begin{aligned}
4 \eta\left(\operatorname{Im} H\left(p_{1}, p_{2}\right)\right) & =\phi\left(p_{2}, p_{1}\right)-\phi\left(p_{1}, p_{2}\right), \\
\mu(p, q) & =-\eta(q) p,
\end{aligned}
$$

for all $p, p_{1}, p_{2} \in \mathbb{C}^{n}, q \in \mathbb{R}^{k}$.

We set

$$
B(p, s):=\eta(s) p, \quad r\left(s_{1}, s_{2}\right):=\frac{1}{2} \nu\left(s_{2}\right) s_{1},
$$

$p \in \mathbb{C}^{n}, s, s_{1}, s_{2} \in \mathbb{C}^{k}$, where $\eta, \nu$ are complex-linearly extended to $\mathbb{C}^{k}$. Then (1.5.b) implies

$$
\operatorname{Re} H(B(p, q), p)=r(H(p, p), q),
$$

for all $p \in \mathbb{C}^{n}, q \in \mathbb{R}^{k}$, which is analogous to (0.2.c). It is follows from (1.6) that $\phi$ is uniquely determined by $\mu$ (as in (1.4) above). Therefore, by (1.5.a) and (1.7.b), $X_{2}$ is uniquely determined by $B$ (note that $B$ also uniquely determines $r$ by (1.8)). Thus, it is clear from $(0.2 . \mathrm{d})$ that to prove the lemma, we need to show that

$$
\operatorname{Im} H(B(p, H(p, p)), p)=0,
$$

for all $p \in \mathbb{C}^{n}$ and that $r\left(s_{1}, s_{2}\right)$ is symmetric. We write $\phi$ in the most general form 


$$
\phi\left(p_{1}, p_{2}\right)=M p_{1} p_{2}+N \overline{p_{1}} p_{2}+T p_{1} \overline{p_{2}}+S \overline{p_{1} p_{2}}
$$

Then (1.5.a), (1.6), (1.7) give

$$
\begin{aligned}
M & =0, \\
S & =0 \\
N \overline{p_{1}} p_{2} p_{3} & =\sqrt{p_{1}} p_{3} p_{2}, \\
T \overline{p_{1}} p_{2} p_{3}-T p_{2} \overline{p_{1}} p_{3} & =2 i \mu\left(p_{3}, H\left(p_{2}, p_{1}\right)\right), \\
T p_{1} \overline{p_{2}} p_{3} & =2 i \mu\left(p_{1}, H\left(p_{3}, p_{2}\right)\right), \\
H\left(N \overline{p_{1}} p_{2} p_{3}, p_{4}\right) & =2 i H\left(p_{2}, \mu\left(p_{1}, H\left(p_{3}, p_{4}\right)\right)\right)-H\left(p_{3}, T p_{1} \overline{p_{2}} p_{4}\right),
\end{aligned}
$$

for all $p_{1}, p_{2}, p_{3}, p_{4} \in \mathbb{C}^{n}$, where $\mu$ is extended in the last argument to a complex-linear mapping on $\mathbb{C}^{k}$. Calculating $\operatorname{Im} H(\eta(H(p, p)) p, p)$ from (1.7.a) we get

$$
\operatorname{Im} H(\eta(H(p, p)) p, p)=\frac{1}{2} \operatorname{Re} H(N \overline{p p} p-T p \overline{p p}, p) .
$$

On the other hand, (1.5.a), (1.6.b), (1.7.b) give

$$
\operatorname{Im} H(\eta(H(p, p)) p, p)=-\frac{1}{2} \operatorname{Re} H(N \bar{p} p p+T p \bar{p} p+M p p p+S \overline{p p} p, p) .
$$

Comparing the last two expressions and using (1.10.a), (1.10.b), (1.10.d), (1.10.e) yields (1.9).

To show that $r\left(s_{1}, s_{2}\right)$ is symmetric, by $(1.5 . \mathrm{b})$, we need to prove that

$$
\operatorname{Re} H\left(\eta\left(H\left(p_{1}, p_{1}\right)\right) p_{2}, p_{2}\right)=\operatorname{Re} H\left(\eta\left(H\left(p_{2}, p_{2}\right)\right) p_{1}, p_{1}\right) \text {, }
$$

for all $p_{1}, p_{2} \in \mathbb{C}^{n}$. It follows from $(1.7 \mathrm{a})$ that

$$
\operatorname{Re} H\left(\eta\left(H\left(p_{1}, p_{1}\right)\right) p_{2}, p_{2}\right)=-\frac{1}{2} \operatorname{Im} H\left(N \overline{p_{1}} p_{1} p_{2}-T p_{1} \overline{p_{1}} p_{2}, p_{2}\right),
$$

for all $p_{1}, p_{2} \in \mathbb{C}^{n}$. On the other hand, (1.5.a), (1.6.b), (1.7.b), (1.10.a), $(1.10 . \mathrm{b})$ give

$$
\operatorname{Re} H\left(\eta\left(H\left(p_{1}, p_{1}\right)\right) p_{2}, p_{2}\right)=-\frac{1}{2} \operatorname{Im} H\left(N \overline{p_{2}} p_{2} p_{1}-T p_{2} \overline{p_{2}} p_{1}, p_{1}\right)
$$

for all $p_{1}, p_{2} \in \mathbb{C}^{n}$, which together with (1.12) implies (1.11).

The lemma is proved.

\section{Lemma 1.3. $\tilde{\mathfrak{g}}_{H}^{3}=\{0\}$.}

Proof. Let $X_{3} \in \tilde{\mathfrak{g}}_{H}^{3}$. Then there exist real-trilinear mappings $\phi\left(\cdot,,^{\circ}\right)$, $\phi\left(\cdot,,^{\circ}\right)$ from $\mathbb{C}^{n} \times \mathbb{C}^{n} \times \mathbb{C}^{n}$ to the spaces $M(n, \mathbb{C})$ and $M(k, \mathbb{R})$ respectively, real-bilinear mappings $\eta(\cdot, \cdot), \nu(\cdot, \cdot)$ from $\mathbb{C}^{n} \times \mathbb{R}^{k}$ to the above spaces of matrices respectively, and a real-trilinear mapping $\mu(\cdot, \cdot, \cdot)$ from $\mathbb{C}^{n} \times \mathbb{C}^{n} \times \mathbb{R}^{k}$ 
to $\mathbb{C}^{n}$ such that

$$
\begin{aligned}
& X_{3}\left(p_{1} \frac{\partial}{\partial z}+2 i H\left(z, p_{1}\right) \frac{\partial}{\partial w}\right)\left(p_{2} \frac{\partial}{\partial z}+2 i H\left(z, p_{2}\right) \frac{\partial}{\partial w}\right)\left(p_{3} \frac{\partial}{\partial z}+2 i H\left(z, p_{3}\right) \frac{\partial}{\partial w}\right)= \\
& \phi\left(p_{1}, p_{2}, p_{3}\right) z \frac{\partial}{\partial z}+\phi\left(p_{1}, p_{2}, p_{3}\right) w \frac{\partial}{\partial w}, \\
& {\left[X_{3}\left(p_{1} \frac{\partial}{\partial z}+2 i H\left(z, p_{1}\right) \frac{\partial}{\partial w}\right)\left(p_{2} \frac{\partial}{\partial z}+2 i H\left(z, p_{2}\right) \frac{\partial}{\partial w}\right)\right]^{\prime}\left(q \frac{\partial}{\partial w}\right)=} \\
& \mu\left(p_{1}, p_{2}, q\right) \frac{\partial}{\partial z}+2 i H\left(z, \mu\left(p_{1}, p_{2}, q\right)\right) \frac{\partial}{\partial w}, \\
& {\left[X_{3}\left(p \frac{\partial}{\partial z}+2 i H(z, p) \frac{\partial}{\partial w}\right)\right]^{\prime}\left(q \frac{\partial}{\partial w}\right)=} \\
& \eta(p, q) z \frac{\partial}{\partial z}+\nu(p, q) w \frac{\partial}{\partial w},
\end{aligned}
$$

$p, p_{1}, p_{2}, p_{3} \in \mathbb{C}^{n}, q \in \mathbb{R}^{k}$, where $\left[X_{3}\left(p_{1} \frac{\partial}{\partial z}+2 i H\left(z, p_{1}\right) \frac{\partial}{\partial w}\right)\left(p_{2} \frac{\partial}{\partial z}+2 i H\left(z, p_{2}\right) \frac{\partial}{\partial w}\right)\right]^{\prime}$ corresponds to $X_{3}\left(p_{1} \frac{\partial}{\partial z}+2 i H\left(z, p_{1}\right) \frac{\partial}{\partial w}\right)\left(p_{2} \frac{\partial}{\partial z}+2 i H\left(z, p_{2}\right) \frac{\partial}{\partial w}\right)$ as an element of $\tilde{\mathrm{g}}_{H}^{1}$ and $\left[X_{3}\left(p \frac{\partial}{\partial z}+2 i H(z, p) \frac{\partial}{\partial w}\right)\right]^{\prime}$ corresponds to $X_{3}\left(p \frac{\partial}{\partial z}+2 i H(z, p) \frac{\partial}{\partial w}\right)$ as an element of $\widetilde{\mathfrak{g}}_{H}^{2}$. Let $X_{3}^{\prime}$ be the corresponding linear mapping from $\mathrm{g}_{H}^{-2}$ to $\tilde{\mathrm{g}}_{H}^{1}$. Then there exist real-bilinear mappings $\lambda(\bullet, \circ)$ and $\rho\left(\bullet,{ }^{\circ}\right)$ from $\mathbb{C}^{n} \times \mathbb{R}^{k}$ to the spaces $M(n, \mathbb{C})$ and $M(k, \mathbb{R})$ respectively such that

$$
X_{3}^{\prime}\left(q \frac{\partial}{\partial w}\right)\left(p \frac{\partial}{\partial z}+2 i H(z, p) \frac{\partial}{\partial w}\right)=\lambda(p, q) z \frac{\partial}{\partial z}+\rho(p, q) w \frac{\partial}{\partial w},
$$

$p \in \mathbb{C}^{n}, q \in \mathbb{R}^{k}$. Equation (0.2.a) gives

$$
\begin{aligned}
\psi\left(p_{1}, p_{2}, p_{3}\right) H(z, z) & =2 \operatorname{Re} H\left(\phi\left(p_{1}, p_{2}, p_{3}\right) z, z\right), \\
\nu(p, q) H(z, z) & =2 \operatorname{Re} H(\eta(p, q) z, z), \\
\rho(p, q) H(z, z) & =2 \operatorname{Re} H(\lambda(p, q) z, z),
\end{aligned}
$$

for all $p, p_{1}, p_{2}, p_{3}, z \in \mathbb{C}^{n}, q \in \mathbb{R}^{k}$. Next, analogously to (1.6), we have

$$
\begin{aligned}
4 \mu\left(p_{1}, p_{2}, \operatorname{Im} H\left(p_{3}, p_{4}\right)\right) & =\phi\left(p_{1}, p_{2}, p_{3}\right) p_{4}-\phi\left(p_{1}, p_{2}, p_{4}\right) p_{3}, \\
4 \operatorname{Im} H\left(\mu\left(p_{1}, p_{2}, q\right), p_{3}\right) & =\phi\left(p_{1}, p_{2}, p_{3}\right) q,
\end{aligned}
$$

for all $p_{1}, p_{2}, p_{3} \in \mathbb{C}^{n}, q \in \mathbb{R}^{k}$. Further, there are the following analogues of identities (1.7)

$$
\begin{aligned}
4 \eta\left(p_{1}, \operatorname{Im} H\left(p_{2}, p_{3}\right)\right) & =\phi\left(p_{1}, p_{3}, p_{2}\right)-\phi\left(p_{1}, p_{2}, p_{3}\right), \\
\mu\left(p_{1}, p_{2}, q\right) & =-\eta\left(p_{1}, q\right) p_{2},
\end{aligned}
$$

for all $p_{1}, p_{2} \in \mathbb{C}^{n}, q \in \mathbb{R}^{k}$. Finally, conditions $(0.6)$ for $L=3$ are equivalent to

$$
\begin{aligned}
4 \lambda\left(p_{1}, \operatorname{Im} H\left(p_{2}, p_{3}\right)\right) & =\phi\left(p_{3}, p_{2}, p_{1}\right)-\phi\left(p_{2}, p_{3}, p_{1}\right), \\
\lambda(p, q) & =\eta(p, q),
\end{aligned}
$$


for all $p, p_{1}, p_{2}, p_{3} \in \mathbb{C}^{n}, q \in \mathbb{R}^{k}$.

We will now show that identities $(1.13)-(1.16)$ imply that $X_{3}=0$. It follows by the argument in Lemma 1.2 from (1.13.a) and identities (1.14), (1.15) that $\phi$ has the form (see $(1.10 . \mathrm{a}),(1.10 . \mathrm{b})$ )

$$
\phi\left(p_{1}, p_{2}, p_{3}\right)=G p_{1} \overline{p_{2}} p_{3}+K \overline{p_{1} p_{2}} p_{3}+F p_{1} p_{2} \overline{p_{3}}+L \overline{p_{1}} p_{2} \overline{p_{3}},
$$

and (see $(1.10 . c)-(1.10 . f))$

$$
\begin{aligned}
& G p_{1} p_{2} p_{3} p_{4}=G p_{1} p_{2} p_{4} p_{3}, \\
& \left(G p_{1}+K \overline{p_{1}}\right) \overline{p_{2}} p_{3} p_{4}-\left(F p_{1}+L \overline{p_{1}}\right) p_{3} \overline{p_{2}} p_{4}=2 i \mu\left(p_{1}, p_{4}, H\left(p_{3}, p_{2}\right)\right), \\
& \left(F p_{1}+L \overline{p_{1}}\right) p_{2} \overline{p_{3}} p_{4}=2 i \mu\left(p_{1}, p_{2}, H\left(p_{4}, p_{3}\right)\right), \\
& H\left(\left(G p_{1}+K \overline{p_{1}}\right) \overline{p_{2}} p_{3} p_{4}, p_{5}\right) \\
& \quad=2 i H\left(p_{3}, \mu\left(p_{1}, p_{2}, H\left(\dot{p}_{5}, p_{4}\right)\right)\right)-H\left(p_{4},\left(F p_{1}+L \overline{p_{1}}\right) p_{2} \overline{p_{3}} p_{5}\right), \\
& K p_{1} p_{2} p_{3} p_{4}=K p_{1} p_{2} p_{4} p_{3},
\end{aligned}
$$

for all $p_{1}, p_{2}, p_{3}, p_{4} \in \mathbb{C}^{n}$, where $\mu$ is extended complex-linearly to $\mathbb{C}^{k}$ in the last argument. Further, (1.15.b), (1.16) imply

$$
L \overline{p_{1}} p_{2} \overline{p_{3}} p_{4}-G p_{2} \overline{p_{1}} p_{3} p_{4}=2 i \mu\left(p_{\mathrm{s}}, p_{4}, H\left(p_{2}, p_{1}\right)\right) \text {, }
$$

for all $p_{1}, p_{2}, p_{3}, p_{4}, p_{5} \in \mathbb{C}^{n}$. From $(1.17 . \mathrm{b})-(1.17 . \mathrm{d}),(1.18)$ we obtain

$$
\begin{aligned}
H\left(K \overline{p_{1} p_{2}} p_{3} p_{4}, p_{5}\right) & =H\left(p_{3}, G p_{5} \overline{p_{4}} p_{1} p_{2}\right)-H\left(p_{4}, F p_{1} p_{2} \overline{p_{3}} p_{5}\right) \\
H\left(G p_{1} \overline{p_{2}} p_{3} p_{4}, p_{5}\right) & =-H\left(p_{3}, L \overline{p_{4}} p_{5} \bar{p}_{1} p_{2}\right)-H\left(p_{4}, L \overline{p_{1}} p_{2} \overline{p_{3}} p_{5}\right), \\
F p_{1} p_{2} p_{3} p_{4} & =-G p_{4} p_{3} p_{1} p_{2} \\
L p_{1} p_{2} p_{3} p_{4} & =L p_{3} p_{4} p_{1} p_{2} \\
K p_{1} p_{2} p_{3} p_{4} & =L p_{1} p_{3} p_{2} p_{4}+L p_{1} p_{4} p_{2} p_{3} \\
G p_{1} p_{2} p_{3} p_{4}+G p_{3} p_{2} p_{1} p_{4} & =F p_{3} p_{1} p_{2} p_{4}
\end{aligned}
$$

for all $p_{1}, p_{2}, p_{3}, p_{4}, p_{5} \in \mathbb{C}^{n}$.

We set

$$
\begin{aligned}
D\left(p_{1}, p_{2}, H\left(p_{3}, p_{4}\right)\right) & :=i G p_{3} \overline{p_{4}} p_{2} p_{1} \\
t\left(H\left(p_{1}, p_{2}\right), H\left(p_{3}, p_{4}\right)\right) & :=-\frac{1}{2} L \overline{p_{2}} p_{1} \overline{p_{4}} p_{3}
\end{aligned}
$$

for all $p_{1}, p_{2}, p_{3}, p_{4} \in \mathbb{C}^{n}$. It follows from (1.17.a), (1.18), (1.19.d) and the non-degeneracy of $H$ that (1.20.a) defines a complex-trilinear form $D$ on $\mathbb{C}^{n} \times$ $\mathbb{C}^{n} \times \mathbb{C}^{k}$ symmetric with respect to the first two variables and $(1.20 . \mathrm{b})$ defines a complex-bilinear symmetric form $t$ on $\mathbb{C}^{k} \times \mathbb{C}^{k}$, both valued in $\mathbb{C}^{n}$. It follows from $(1.13 . \mathrm{a}),(1.19 . \mathrm{c}),(1.19 . \mathrm{e})$ that $X_{3}$ is uniquely determined by $t$ (note that it follows from $(1.19 . \mathrm{b})$ that $D$ is uniquely determined by $t$ ). The forms $D$ and $t$ satisfy the following relations

$$
\begin{aligned}
H(D(p, p, q), p) & =4 i H(p, t(q, H(p, p)), \\
H(D(p, p, H(p, p)), p) & =0,
\end{aligned}
$$


for all $p \in \mathbb{C}^{n}, q \in \mathbb{R}^{k}$. Indeed, (1.21.a) follows from (1.19.b), (1.19.d); to prove $(1.21 . b)$ we note that it follows from $(1.19 . c),(1.19 . f)$ that $G p \overline{p p} \equiv 0$.

We will now show that equations $(1.21)$ can have only zero solutions. For this we note that a polynomial vector field $X$ on $\mathbb{C}^{n+k}$ of the form

$$
X=\left(D^{\prime}(z, z, w)+t^{\prime}(w, w)\right) \frac{\partial}{\partial z}+2 i H\left(z, t^{\prime}(\bar{w}, \bar{w})\right) \frac{\partial}{\partial w}
$$

defines an infinitesimal holomorphic automorphism of $Q_{H}$ (i.e. $X \in \mathrm{g}_{H}$ ) if and only if the following conditions are satisfied

$$
\begin{aligned}
H\left(D^{\prime}(z, z, u), z\right) & =4 i H\left(z, t^{\prime}(u, H(z, z))\right), \\
H\left(D^{\prime}(z, z, H(z, z)), z\right) & =0,
\end{aligned}
$$

for all $z \in \mathbb{C}^{n}, u \in \mathbb{R}^{k}$, where $D^{\prime}$ is a complex-trilinear form on $\mathbb{C}^{n} \times \mathbb{C}^{n} \times \mathbb{C}^{k}$ symmetric with respect to the first two variables, and $t^{\prime}$ is a complex-bilinear symmetric form on $\mathbb{C}^{k} \times \mathbb{C}^{k}$, both valued in $\mathbb{C}^{n}$. Since the vector field $X$ has weight 3 , it must be zero by $(0.1)$. This means that equations $(1.22)$ can have only zero solutions and therefore equations (1.21) have only zero solutions also.

Thus, $X_{3}=0$, and the lemma is proved.

\section{References}

[1] Beloshapka, V., A uniqueness theorem for automorphisms of a nondegenerate surface in a complex space (translated from Russian), Math. Notes, 47 (1990), 239-242.

[2] Cartan, É., Sur la géometrie pseudo-conforme des hypersurfaces de l'espace de deux variables complexes: I, Ann. Math. Pura Appl. 11 (1932), 17-90; II, Ann. Scuola Norm. Sup. Pisa, 1 (1932), 333-354.

[3] Chern, S. S. and Moser, J. K., Real hypersurfaces in complex manifolds, Acta Math, 133 (1974), 219-271.

[4] Ezhov, V., Isaev, A. and Schmalz, G., Invariants of elliptic and hyperbolic $C R$-structures of codimension 2, Internat. J. Math., to appear.

[5] Ezhov, V. and Schmalz, G., Holomorphic automorphisms of nondegenerate CR-quadrics: explicit description, J. Geom. Analysis, to appear.

[6] A simple proof of Beloshapka's theorem on parametrisation of the automorphism groups of $C R$-manifolds (Russian), Mat. Zametki, to appear.

[7] Forstnerič, F., Mappings of quadric Cauchy-Riemann manifolds, Math. Ann., 292 (1992), 163-180.

[8] Khenkin, G., Tumanov, A., Local characterization of holomorphic automorphisms of Siegel domains (translated from Russian), Funct. Anal. Appl., 17 (1983), 285-294.

[9] Lai, H.-F., Real submanifolds of codimension two in complex manifolds, Trans. Amer. Math. Soc., 264 (1981), 331-352.

[10] Satake, I., Algebraic Structures of Symmetric Domains, Kanô Memorial Lectures 4, Iwanami Shoten, Tokyo; Princeton University Press, 1980.

[11] Tanaka, N., On generalized graded Lie algebras and geometric structures I, J. Math. Soc. 
Japan, 19 (1967), 215-254.

[12] Tumanov, A., Finite-dimensionality of the group of $C R$ automorphisms of a standard $C R$ manifold, and proper holomorphic mappings of Siegel domains (translated from Russian), Math. USSR. Izv., 32 (1989), 655-662. 
